\title{
Gamma-ray observations of supernova remnants
}

\author{
Marianne Lemoine-Goumard \\ Centre d'Études Nucléaires de Bordeaux Gradignan \\ Université Bordeaux 1, CNRS/IN2P3 \\ 33175 Gradignan, France \\ E-mail: lemoine@cenbg.in2p3.fr \\ Funded by contract ERC-StG-259391 from the European Community
}

\begin{abstract}
In the past few years, gamma-ray astronomy has entered a golden age. At TeV energies, only a handful of sources were known a decade ago, but the current generation of ground-based imaging atmospheric Cherenkov telescopes has increased this number to more than one hundred. At GeV energies, the Fermi Gamma-ray Space Telescope has increased the number of known sources by nearly an order of magnitude in its first 2 years of operation. The recent detection and unprecedented morphological studies of gamma-ray emission from shelltype supernova remnants is of great interest, as these analyses are directly linked to the long standing issue of the origin of the cosmic-rays. However, these detections still do not constitute a conclusive proof that supernova remnants accelerate the bulk of Galactic cosmic-rays, mainly due to the difficulty of disentangling the hadronic and leptonic contributions to the observed gammaray emission. In the following, I will review the most relevant results of gamma ray astronomy concerning supernova remnants (shell-type and middle-age interacting with molecular clouds).
\end{abstract}

Keywords. cosmic-rays; supernova remnants; gamma-rays

\section{The cosmic-ray mystery}

\subsection{The link between cosmic-rays and supernova remnants}

The association between supernova remnants (SNRs) and Galactic cosmic rays (CRs) is very popular since 1934, when Baade and Zwicky argued that this class of astrophysical objects can account for the required CR energetics (W. Baade and F. Zwicky (1934)). Indeed, in order to maintain the cosmic-ray energy density in the Galaxy, about 3 supernovae per century should transform 10 percent of their kinetic energy in cosmic-ray energy. This argument has also been supported by E. Fermi's proposal of a very general mechanism for particle acceleration, which is very efficient if applied at SNR shocks (A. R. Bell (1978)). The extremely interesting point of the diffusive shock acceleration (DSA) mechanism is that it naturally yields power-law spectra for the energy distribution of accelerated particles. However, until recently there were absolutely no observational evidence concerning the acceleration of protons and nuclei in SNRs. Indeed, through their interaction with the interstellar magnetic fields, the charged particles arriving on Earth have lost all directional information and cannot be used to pinpoint the sources. That is why, almost 100 years after their discovery by V. Hess, the origins of the cosmic-rays and their cosmic accelerators remain unknown.

Astronomy with gamma-rays provides a means to study these sources of high energy particles. Indeed, cosmic rays (ionized nuclei of all species, but mostly protons, plus a small fraction of electrons) can interact with ambient matter and photons producing gamma-rays via two different channels. One mechanism invokes the interaction of 
accelerated protons at supernova remnants shocks with interstellar material generating neutral pions which in turn decay into gamma rays. We call this mechanism the hadronic scenario. A second competing channel exists in the inverse Compton scattering of the photon fields in the surroundings of the SNR by the same relativistic electrons that generate the synchrotron X-ray emission. This is the leptonic scenario. Being of leptonic or hadronic origin, these gamma-rays are not affected while they travel to Earth and can therefore be used to pinpoint the cosmic accelerators in our Galaxy.

\subsection{Gamma-ray experiments}

Two major breakthroughs occurred recently in gamma-ray astronomy. Firstly, after more than 20 years of development, the first source of very high energy gamma-rays, the Crab Nebula, was discovered in 1989 by the Whipple telescope. Since this date the technical progresses in this field have led to important scientific results, especially by the Cherenkov telescopes H.E.S.S., VERITAS and MAGIC. These ground-based experiments for gamma-ray astronomy rely on the development of cascades (air-showers) initiated by astrophysical gamma-rays. Such cascades only persist to ground-level above $1 \mathrm{TeV}$ and only produce significant Cherenkov light above a few $\mathrm{GeV}$, setting a fundamental threshold to the range of this technique. Today, more than 160 gamma-ray sources have been detected with high significance, 23 being associated to supernova remnants or molecular clouds.

Second, in space, the Large Area Telescope (LAT) onboard the Fermi satellite has considerably improved our knowledge of the $0.1-100 \mathrm{GeV}$ gamma-ray sky with 1873 objects detected in only two years of observation (P. L. Nolan et al. (2012)). It has moved the field from the detection of a small number of sources to the detailed study of several classes of Galactic and extragalactic objects. A complete study of association of the 1873 sources detected show that $\sim 4 \%$ of them are associated to supernova remnants (P. L. Nolan et al. (2012)).

Thanks to the observations of radio and X-ray synchrotron from a large number of supernova remnants, there is no doubt today that supernova remnants can accelerate efficiently particles up to $10^{14} \mathrm{eV}$. The question is whether these particles are protons or electrons and if they can be accelerated up to the knee of the cosmic-ray spectrum $\left(10^{15} \mathrm{eV}\right)$. A direct signature of accelerated protons is expected through pion decay emission in the $\mathrm{GeV}-\mathrm{TeV}$ gamma ray range.

\section{Detection of supernova remnants in gamma-rays}

The sample of supernova remnants detected in gamma-rays is now extremely large: it goes from evolved supernova remnants interacting with molecular clouds (MC) up to young shell-type supernova remnants and historical supernova remnants. The Fermi-LAT even detected one evolved supernova remnant without MC interaction, Cygnus loop. This section will review some of the main characteristics of detected SNRs. Complementary informations on GeV SNRs can be found in the description of Puppis A (J. W. Hewitt et al. (2012)) and in the Fermi Catalog of Supernova Remnants (T. J. Brandt (2013)).

\subsection{Historical supernova remnants}

Multi-PeV protons can be accelerated only during a relatively short period of the SNR evolution, namely, at the end of the free-expansion phase/beginning of the Sedov phase, when the shock velocity is high enough to allow sufficiently high acceleration rate. When the SNR enters the Sedov phase, the shock slows down leading to a decrease of the 
maximum energy of the particles that can be confined within the SNR. Therefore, historical supernova remnants are excellent targets for gamma-ray observations.

Two historical SNRs have been detected both at $\mathrm{GeV}$ and $\mathrm{TeV}$ energies: Cassiopeia A (Cas A) (A. A. Abdo et al. (2010), J. Albert et al. (2007), V. A. Acciari et al. (2010)) and Tycho (F. Giordano et al. (2012), V. A. Acciari et al. (2011)).

Cas A is the remnant of SN 1680. It is the brightest radio source in our Galaxy and its overall brightness across the electromagnetic spectrum makes it a unique laboratory for studying high-energy phenomena in SNRs. A multiwavelength modeling of Cas A does not allow a discrimination between the hadronic and leptonic scenarios. However, regardless of the origin of the observed gamma rays, this modeling implies that the total content of CRs accelerated in Cas $\mathrm{A}$ is $\sim(1-2) \times 10^{49} \mathrm{erg}$, and the magnetic field amplified at the shock can be constrained as $\mathrm{B} \approx 0.12 \mathrm{mG}$. Even though Cas $\mathrm{A}$ is considered to have entered the Sedov phase, the total amount of CRs accelerated in the remnant constitutes only a minor fraction $(\sim 2 \%)$ of the total kinetic energy of the supernova, which is well below the $\sim 10 \%$ commonly used to maintain the cosmic-ray energy density in the Galaxy.

Tycho's SNR (SN 1572) is classified as a Type Ia (thermonuclear explosion of a white dwarf) based on observations of the light-echo spectrum. Thanks to the large amount of data available at various wave bands, this remnant can be considered one of the most promising object where to test the shock acceleration theory and hence the CR - SNR connection. First, using the precise radio and X-ray observations of this SNR, G. Morlino \& D. Caprioli (2012) have shown that the magnetic field at the shock has to be $>200 \mu \mathrm{G}$ to reproduce the data. Then, using multiwavenlength data, especially the $\mathrm{GeV}$ and $\mathrm{TeV}$ detections, they could infer that the gamma-ray emission detected from Tycho cannot be of leptonic origin, but has to be due to accelerated protons (this result is consistent with another modeling proposed in F. Giordano et al. (2012)). These protons are accelerated up to energies as large as $\sim 500 \mathrm{TeV}$, with a total energy converted into CRs estimated to be about $12 \%$ of the forward shock bulk kinetic energy. This is much more reasonable in the context of acceleration of Galactic cosmic-rays in SNRs.

\subsection{Young shell-type supernova remnants}

Four young shell-like SNRs with clear shell-type morphology resolved in VHE gammarays have been detected by H.E.S.S.: RX J1713.7-3946 (F. Aharonian et al. (2004), F. Aharonian et al. (2005)), RX J0852.04622 - also known as Vela Junior - (F. Aharonian et al. (2007)), SN 1006 (F. Acero et al. (2010)) and HESS J1731-347 (A. Abramowski et al. (2011)). A fifth case, RCW 86 (F. Aharonian et al. (2011)), might be added to this list although the $\mathrm{TeV}$ shell morphology has not yet been clearly proved. Two of them, RX J1713.7-946 (A. A. Abdo et al. (2011)) and Vela Junior (T. Tanaka et al. (2011)), have been detected by Fermi-LAT allowing direct investigation of young shell-type SNRs as sources of cosmic rays. Concerning RX J1713.7-3946, the Fermi-LAT spectrum is well described by a very hard power-law with a photon index of $\Gamma=1.5 \pm 0.1$ that coincides in normalization with the steeper H.E.S.S.-detected gamma-ray spectrum at higher energies. The GeV measurements with Fermi-LAT do not agree with the expected fluxes around $1 \mathrm{GeV}$ in most hadronic models published so far (e.g., E. G. Berezhko \& H. J. Voelk (2010)) and requires an unrealistically large density of the medium. The agreement with the expected IC spectrum is better (as can be seen in Figure 1) but requires a very low magnetic field of $\sim 10 \mu \mathrm{G}$ in comparison to the one measured in the thin filaments by $\mathrm{X}$-ray observations. It is possible to reconcile a high magnetic field with the leptonic model if $\mathrm{GeV}$ gamma rays are radiated not only from the filamentary structures seen by Chandra, but also from other regions in the SNR where the magnetic field may be 

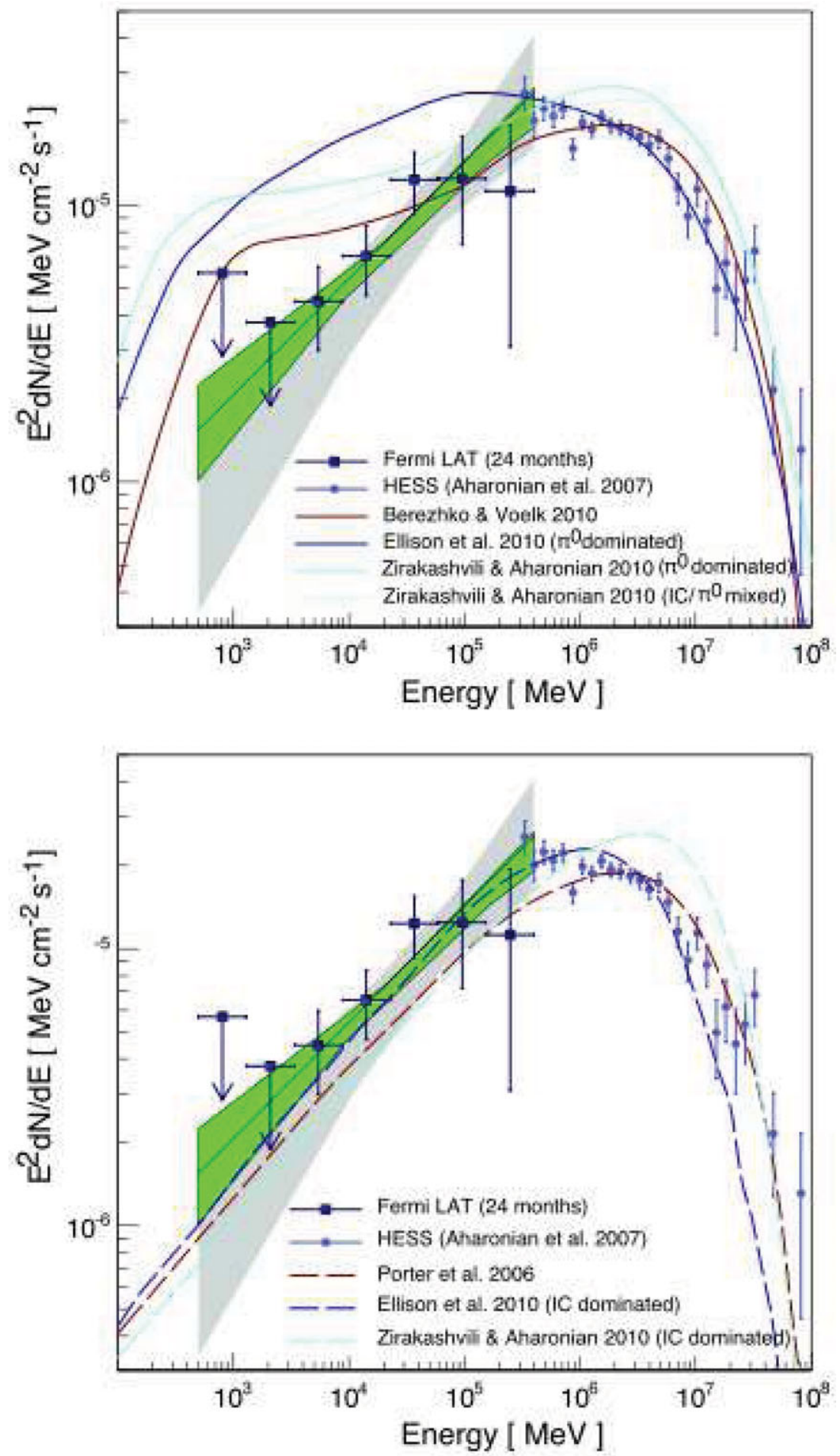

Figure 1. Energy spectrum of RX J1713.7-3946 in gamma rays. Shown is the Fermi-LAT (A. A. Abdo et al. (2011)) detected emission in combination with the energy spectrum detected by H.E.S.S. (F. Aharonian et al. (2005)). See A. A. Abdo et al. (2011) for more details. 
weaker. Similar conclusions are reported for Vela Junior supernova remnant even though in this case the hadronic scenario can not be ruled out. However, being of hadronic or leptonic origin, the $\mathrm{GeV}-\mathrm{TeV}$ gamma-ray detections imply a low maximal energy for the accelerated particles of $\sim 100 \mathrm{TeV}$, well below the knee of the cosmic-ray spectrum.

\subsection{Supernova remnants interacting with molecular clouds}

The Fermi LAT Collaboration has reported the discoveries of several middle aged $\left(\sim 10^{4}\right.$ yrs) remnants interacting with molecular clouds: W51C (A. A. Abdo et al. (2010)), W44 (A. A. Abdo et al. (2010)), IC 443 (A. A. Abdo et al. (2010)), W49 (A. A. Abdo et al. (2010)) and W28 (A. A. Abdo et al. (2010)), being the most famous cases. Apart from W44, they have all been detected in the TeV regime as well. These SNRs are generally much brighter in $\mathrm{GeV}$ than in $\mathrm{TeV}$ in terms of energy flux (due to a spectral steepening arising at a few $\mathrm{GeV}$ ), which emphasizes the importance of the GeV observations. The interaction with a molecular cloud provides the target material that allows to enhance the gamma-ray emission, either through bremsstrahlung by relativistic electrons or by pion-decay gamma-rays produced by high-energy protons. The observed large luminosity of the GeV gamma-ray emission precludes the inverse-Compton scattering off the CMB and interstellar radiation fields as the main emission mechanism since it would require an extremely low density (to suppress the bremsstrahlung and proton-proton interaction), a low magnetic field to enhance the gamma/X-ray flux ratio and an unrealistically large energy injected into protons. In addition, the break in the electron spectrum corresponding to the gamma-ray spectrum directly appears in the radio data leading to a bad modeling of the radio data and therefore disfavors the bremsstrahlung process. A model in which gamma-rays are produced via proton-proton interaction gives the most satisfactory explanation for the GeV gamma-rays observed in SNRs interacting with molecular gas as seen in Figure 2 for the case of W51C. However, the hadronic nature of the gamma-ray signal is mostly inferred from indirect arguments in such cases. Recently, an improved spectral analysis of W44 and IC 443 have been carried using four years of Fermi-LAT data above $60 \mathrm{MeV}$ M. Ackermann et al. (2013). Both spectra are steeply rising below $200 \mathrm{MeV}$, showing a clear break at around $200 \mathrm{MeV}$ (see the case of IC 443 in Figure 3). This spectral feature is often referred to as the "pion-decay bump" and uniquely identifies pion-decay gamma-rays. This detection thus provides a direct evidence that cosmic-ray protons are accelerated in SNRs W44 and IC 443.

There are two different types of hadronic scenarios to explain the GeV gamma-ray emission arising from such SNRs: the "Runaway CR" model (F. Aharonian \& A. M. Atoyan (1996), Y. Ohira, K. Murase \& R. Yamazaki (2011)) and the "Crushed Cloud" model (Y. Uchiyama et al. (2011)). The Runaway CR model considers gamma-ray emission from molecular clouds illuminated by runaway CRs that have escaped from their accelerators, whereas the Crushed Cloud model invokes a shocked molecular cloud into which cosmic-ray particles are adiabatically compressed and accelerated resulting in enhanced synchrotron and pion-decay gamma-ray emissions.

\section{Where are the PeVatrons ?}

The recent $\mathrm{GeV}$ and $\mathrm{TeV}$ detections of supernova remnants confirm the theoretical predictions that supernova remnants can operate as powerful cosmic ray accelerators. However, if these objects are responsible for the bulk of galactic cosmic rays, they should be able to accelerate protons and nuclei at least up to $10^{15} \mathrm{eV}$ and therefore act as PeVatrons. S. Gabici \& F. A. Aharonian (2007) have shown that the spectrum of nonthermal particles extends to $\mathrm{PeV}$ energies only during a relatively short period of the evolution of 


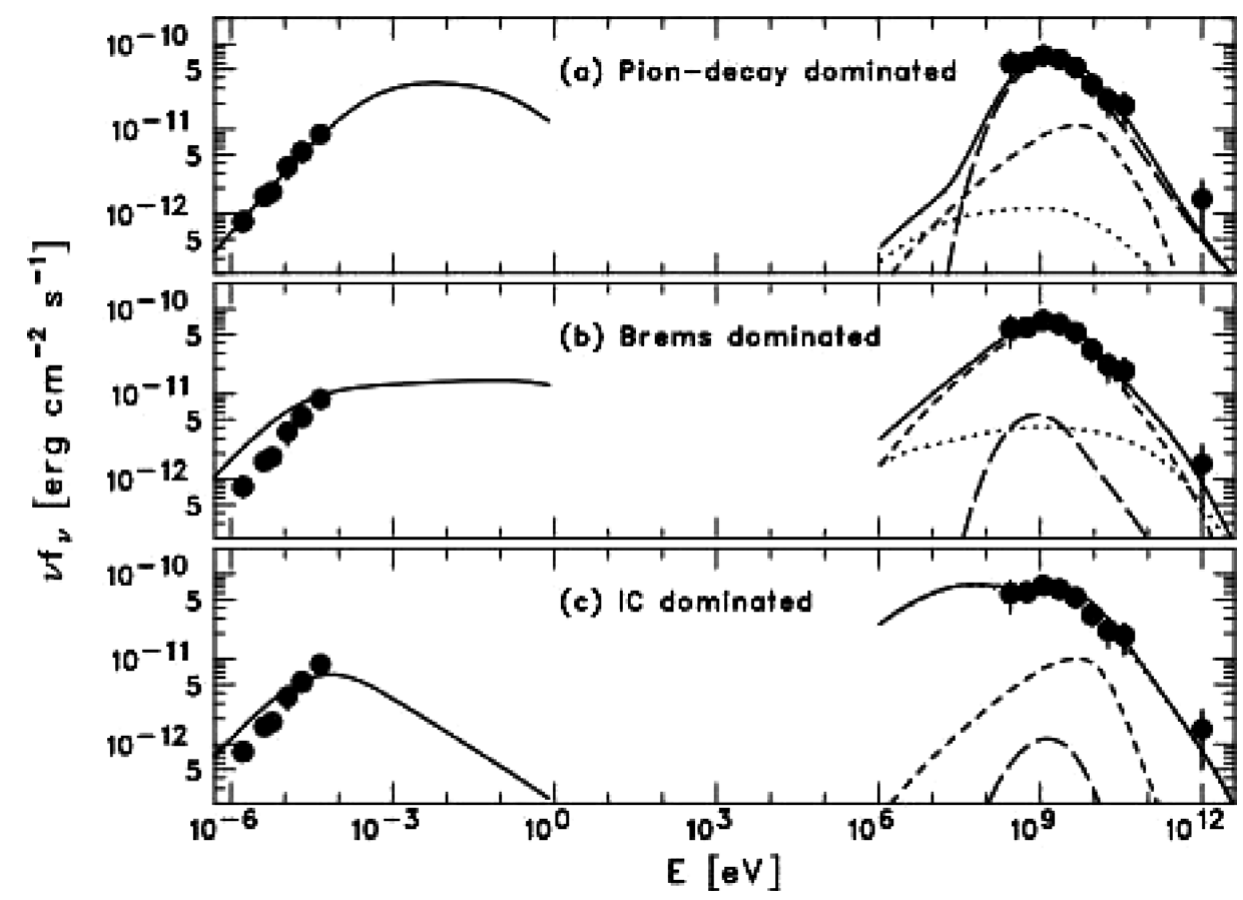

Figure 2. Different scenarios proposed for the multiwavelength modeling of W51C A. A. Abdo et al. (2010). The radio emission (from Moon \& Koo 1994) is explained by synchrotron radiation, while the gamma-ray emission is modeled by different combinations of pion-decay (long-dashed curve), bremsstrahlung (dashed curve), and IC scattering (dotted curve). The sum of the three component is shown as a solid curve. See A. A. Abdo et al. (2010) for more details.

the remnant since high energy particles are the first to escape from the supernova remnant shock. For this reason one may expect spectra of secondary gamma-rays extending to energies beyond $10 \mathrm{TeV}$ only from less than $1 \mathrm{kyr}$ old supernova remnants. In this respect, Tycho could be considered as a half-PeVatron at least, since there is no evidence of a cut-off in the VERITAS data. One may wonder how many PeVatrons are expected to be detectable in our Galaxy. A simple estimate has been provided by Gabici and Aharonian (2007): assuming a rate of $\sim 3$ supernovae per century in our Galaxy, this directly implies that only a dozen of PeVatrons are present in the Galaxy on average and hence that they are likely to be distant and weak. This emphasizes the importance of $\mathrm{TeV}$ observations by the future generation of Cherenkov telescopes such as the Cherenkov Telescope Array (CTA) which will have a better effective area in the energy range already covered but that will also allow the observation up to $100 \mathrm{TeV}$ of sources such as Tycho, therefore constraining the maximal energy at which protons are being accelerated in young SNRs. Monte-Carlo simulations of shell-type SNRs have already been carried out for different CTA array layouts, assuming a uniform exposure time of $20 \mathrm{hr}$ everywhere along the Galactic Plane. In this purpose, the morphological and spectral characteristics of three SNRs (RX J1713.7-3946, Vela Junior and RCW 86), as measured with H.E.S.S., together with their respective distance estimates, have been used to simulate sources throughout the inner Galaxy (F. Acero et al. (2013), M. Renaud (2011)). This leads to $\sim 20-70$ detectable TeV SNRs, among which $\sim 7-15$ would be resolved with CTA (configurations I and $\mathrm{D}$, optimized for providing the best sensitivity over the whole energy range or above $1 \mathrm{TeV}$, respectively). It is stricking to note that, thanks to its increased sensitivity, CTA will have the capability to detect SNRs as luminous as RX J1713.7-3946, Vela Junior, 


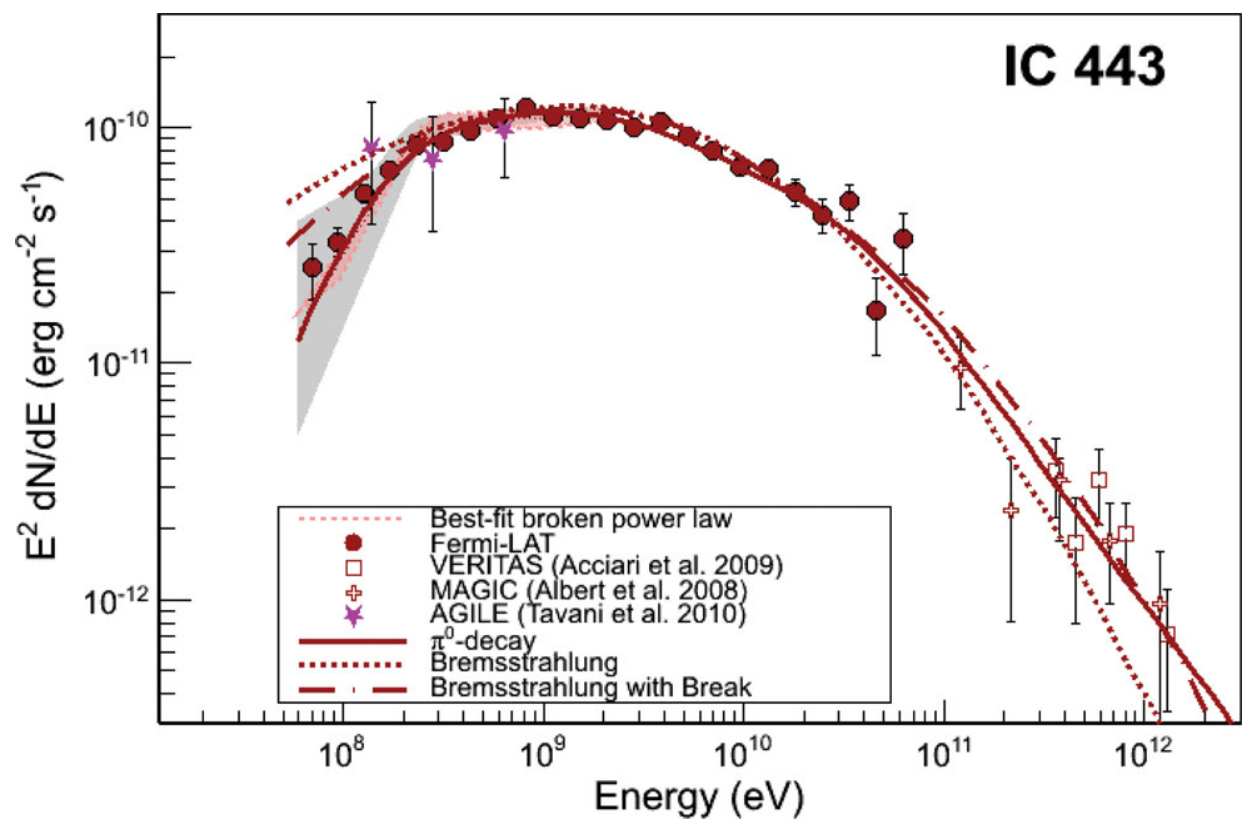

Figure 3. Gamma-ray spectra of IC 443 as measured with the Fermi-LAT. Color-shaded areas bound by dashed lines denote the best-fit broadband smooth broken power law $(60 \mathrm{MeV}$ to $2 \mathrm{GeV}$ ), gray-shaded bands show systematic errors below $2 \mathrm{GeV}$ due mainly to imperfect modeling of the galactic diffuse emission. At the high-energy end, TeV spectral data points from MAGIC and VERITAS are shown. Solid lines denote the best-fit pion-decay gamma-ray spectra, dashed lines denote the best-fit bremsstrahlung spectra, and dash-dotted lines denote the best-fit bremsstrahlung spectra when including an ad hoc low-energy break at $300 \mathrm{MeV} c^{-1}$ in the electron spectrum. See M. Ackermann et al. (2013) for more details.

or RCW 86 up to the other side of the Galaxy, providing a complete population study of our Galactic cosmic-ray accelerators.

\section{Acknowledgements}

I thank all the members of the Fermi GALACTIC and HESS SNR-PWN working groups for valuable discussion. I gratefully acknowledge funding from the European Community (contract ERC-StG-259391).

\section{References}

A. A. Abdo et al. 2011, ApJ, 734, 28

A. A. Abdo et al. 2010, ApJL, 710, L92

A. A. Abdo et al. 2010, ApJ, 722, 1303

A. A. Abdo et al. 2010, ApJ, 718, 348

A. A. Abdo et al. 2010, ApJ, 712, 459

A. A. Abdo et al. 2010, Science, 327, 1103

A. A. Abdo et al. 2009, ApJL, 706, 1

A. Abramowski et al. 2011, A\& A, 531, A81

F. Acero et al. 2013, Astroparticle Physics, 43, 276

F. Acero et al. 2010, A\&SA, 512, A62

V. A. Acciari et al. 2011, ApJL, 730, L20

V. A. Acciari et al. 2010, ApJ, 714, 163 
M. Ackermann et al. 2013, Science, 339, 807

F. Aharonian et al. 2011, ApJ, 692, 1500

F. Aharonian et al. 2011, A\&SA, 464, 235

F. Aharonian et al. 2005, A\&AA, 437, L7

F. Aharonian et al. 2004, Nature, 432, 75

F. Aharonian \& A. M. Atoyan 1996, A\& $A, 309,917$

J. Albert et al. 2007, A\&3A, 474, 937

W. Baade \& F. Zwicky 1934, Proceedings of the National Academy of Science, 20, 259

A. R. Bell 1978, MNRAS, 182, 147

E. G. Berezhko \& H. J. Voelk 2010, A\&̧A, 511, A34

T. Brandt 2013, these proceedings

S. Gabici \& F. A. Aharonian 2007, ApJ, 665, L131

F. Giordano et al. 2012, ApJL, 744, L2

J. W. Hewitt 2012, ApJ, 759, 89

J. P. Hughes \& C. E. Rakowski and A. Decourchelle 2009, ApJ, 543, L61

G. Morlino \& D. Caprioli 2012, A\& $A$, 538, A81

P. L. Nolan et al. 2012, ApJS, 199, 31

Y. Ohira, K. Murase \& R. Yamazaki 2011, MNRAS, 410, 1577

M. Renaud et al. 2011, CRISM proceedings, Memorie della Societa Astronomica Italiana

T. Tanaka et al. 2011, ApJL, 740, L51

Y. Uchiyama et al. 2010, ApJL, 723, 122

Y. Uchiyama et al. 2007, Nature, 449, 576

\section{Discussion}

WANG: How many super bubbles have been detected as TeV sources?

Lemoine-Goumard: There are two. But their IDs are still very uncertain. 